\title{
Microdissection Testicular Sperm Extraction (micro- TESE) as a Sperm Acquisition Method for Men with Nonobstructive Azoospermia Seeking Fertility: Operative and Laboratory Aspects
}

\author{
Sandro C. Esteves \\ ANDROFERT, Center for Male Reproduction
}

Introduction: Rare foci of sperm production may be found in up to 60\% of men with nonobstructive azoospermia (NOA). Sperm production, if present, is minimal for sperm appearance in the ejaculate. Given that there are no treatment options to restore fertility, sperm retrieval is the only alternative to find testicular sperm than then can be used for in vitro fertilization (IVF). Among sperm acquisition methods, micro-TESE has higher success rates at obtaining sperm compared with testicular sperm extraction and testicular sperm aspiration.

Materials and Methods: This video describes the operative aspects of micro-TESE, performed on an outpatient basis, in a man with NOA and history of cryptorchidism in whom orchidopexy was performed at age 6. The concept of micro-TESE is to identify areas of sperm production within the testes with the aid of optical magnification (15-25X) and based on the size and appearance of the seminiferous tubules (ST).

Results: Intraoperative findings revealed homogeneous pattern of collapsed STs in which an area containing dilated STs was clearly identified and extracted. Testicular tissue was then processed in the IVF laboratory to allow sperm search. In this case, micro-TESE was successful at obtaining testicular sperm for intracytoplasmic sperm injection (ICSI). Surplus retrieved testicular spermatozoa not used for ICSI was cryopreserved. The operative time was 120 minutes, and intraoperative blood loss was negligible. Postoperatively, the patient recovered to his normal activities within 5 days and no complications were recorded except for minor testicular pain and scrotal swelling.

Conclusion: Micro-TESE allowed the identification and extraction of sperm-containing STs with minimum tissue excision and marked reduction in time processing of testicular specimens for sperm injection.

\section{ARTICLE INFO}

www.brazjurol.com.br/videos/may_june_2013/Esteves_440_441video.htm

Int Braz J Urol. 2013; 39 (Video \#8): 440-41

Submitted for publication:

January 10, 2013

Accepted after revision:

February 27, 2013
Correspondence address: Dr. Sandro C. Esteves

ANDROFERT, Center for Male Reproduction 13075-460 - Avenida Dr. Heitor Penteado, 1464

Campinas, SP, Brazil

Phone: +55 19 3295-8877

Fax: +55 $193294-6992$

Email: s.esteves@androfert.com.br 


\section{EDITORIAL COMMENT}

The authors from Sao Paulo, Brazil elegantly demonstrate the operative aspects of microTesticular Sperm Extraction (TESE), performed on a man with nonobstructive azoospermia (NOA). In men with NOA, retrieval of spermatozoa offers an opportunity for fertility despite limited sperm production. This is performed with the aid of optical magnification (25X) and is processed in an in-vitro fertilization laboratory. Micro-TESE is successful at obtaining testicular sperm for intracytoplasmic sperm injection (ICSI). This video nicely portrays the surgical approach of microTESE and the importance of in-vitro fertilization in patients with NOA. While performing microdissection, one can identify seminiferous tubules with sperm and those with Sertoli cells only, which appear atretic. The difference between the larger and smaller tubules is not visible without optical magnification. The use of magnification and processing of the spermatozoa was accurately described by the authors. Since micro-TESE was described, microdissection has shown an improvement of sperm retrieval rate and decrease the amount of tissue excised (1).

\section{REFERENCES}

1. Schlegel PN: Testicular sperm extraction: microdissection improves sperm yield with minimal tissue excision. Hum Reprod. 1999; 14: $131-5$.

Dr. Rafael E. Carrion University of South Florida

Program Chair Department of Urology Division of Andrology

Tampa, FL, 33602, USA

E-mail: rcarrion@health.usf.edu 